California, Irvine, Medical Center, 101 City Drive South, Orange, CA 92868).

COMMENT. Localized hemispheric structural anomalies may occur in children with ADHD, consistent with abnormal frontal-striatal function, and possibly correlated with response to stimulant medication. A neurodevelopmental process is suggested because the hemispheric volumes are similar to controls, and the changes are not associated with ventricular enlargement or the result of atrophy.

Temporal lobe arachnoid cyst - ADHD syndrome. A cerebral developmental anomaly and syndrome is reported in three children with ADHD found on MRI to have left sided temporal lobe arachnoid cysts (Millichap JG. Temporal lobe arachnoid cyst - attention deficit disorder syndrome: Role of the electroencephalogram in diagnosis. Neurology May 1997;48: in press). The diagnosis by MRI was determined following EEGs obtained because of headaches, language delay, or episodes suggestive of partial seizures. EEGs showed focal epileptiform or slow wave abnormalities involving the left temporal region and correlating with the location of the structural temporal fossa lesions. A causal association was considered plausible because of coincidental learning and language disabilities explained by temporal lobe and sylvian region pathology.

\title{
METHYLPHENIDATE EFFECTS ON ATTENTION AND LEARNING
}

The effects of methylphenidate (MPH), in 2 doses $(0.3$ and $0.8 \mathrm{mg} / \mathrm{kg})$, on an attentional continuous performance task (CPT) and on a nonverbal learning task presented in two levels of difficulty, were evaluated in 23 children, aged 7 to 11 years, with attention deficit hyperactivity disorder treated at the Scottish Rite Children's Medical Center, Atlanta, GA. Attention task performance by the group was improved with low-dose MPH compared to placebo; CPT commission errors were reduced, while omission errors were not affected. On nonverbal learning and memory tasks, the easy level of task performance was improved equally with either dose of MPH, whereas the hard task was performed better with the high dose. (O'Toole K, Abramowitz A, Morris R, Dulcan M. Effects of methylphenidate on attention and nonverbal learning in children with attention-deficit hyperactivity disorder. I Am Acad Child Adolesc Psychiatry April 1997;36:531-538). (Reprints: Dr O'Toole, Department of Neuropsychology, Scottish Rite Children's Medical Center, 1001 Johnson Ferry Road, Atlanta, GA 30342).

COMMENT. Low dose methylphenidate may control impulsivity in ADHD children, whereas high doses may be required to improve retention and recall of complex nonverbal information. Cognitive function as well as behavior may be sensitive to stimulant medication, but doses must be individualized and titrated for each patient. Results of group responses may not be applied to all individuals.

Body mass is not predictive of clinical response to MPH, according to a study of 76 children with ADHD receiving doses of 5 to $20 \mathrm{mg}$ at the University of Hawaii, Honolulu, HI. (Rapport MD, Denney C. Titrating methylphenidate in children with attention-deficit/hyperactivity disorder: Is body mass predictive of clinical response? I Am Acad Child Adolesc Psychiatry, April 1997;36:523-530). Dose-response does not differ in children of varying body mass, and the dose of MPH should not be calculated on the basis of body weight. 